\title{
A LINEAR DESCRIPTION OF SHORTENING INDUCED CHANGES IN ISOMETRIC LENGTH-FORCE CHARACTERISTICS OF RAT MUSCLE.
}

\author{
K. Meijer", H.J. Grootenboer", H.F.J.M. Koopman" and P.A. Huijing" s. \\ ' Institute for Biomedical Technology, University of Twente, P.O Box 217, \\ 7500 AE Ensehede, The Netherlands. E-mail: k.meijer@wb.utwente.nl. \\ ${ }^{\$}$ Faculteit Bewegingswetenschappen, Vrije Universteit, Amsterdam, The Netherlands
}

\begin{abstract}
Active muscle shortening reduces the isometric force potential of muscle. This observation indicates that the isometric length-force characteristics are altered during muscle shortening. Post-shortening decrease in isometric force depends on starting length, shortening amplitude and shortening velocity. In the present study, post-shortening decrease in isometric force was determined after isokinetic contractions with various shortening amplitudes initiated from different lengths of rat medial gastrocnemius muscle. For a given target length post-shortening force decrease increased linearly with shortening amplitude. We found that the quotient of post-shortening force decrease and shortening amplitude depended linearly on muscle length. Based on our experimental results and those from other studies, we conclude that shortening induced changes in isometric length-force characteristics can be described by two simple functions. A linear function describing the influence of starting muscle length and shortening amplitude and an exponential function describing the influence of shortening velocity.
\end{abstract}

\section{Introduction}

Many studies have shown that the isometric force potential of the muscle decreases after shortening (e.g. [2]). This decrease depends on muscle length, shortening amplitude and shortening velocity. Post-shortening decrease in isometric force is largest if shortening takes place over optimum muscle length [2]. For a given target muscle length and shortening velocity, a linear dependence of force decrease on shortening amplitude has been found ([1], [2]). These observations indicate that the isometric length-force characteristics of muscle are altered considerably during shortening. A previous study has confirmed this for pennate rat muscle [3].
Isometric length-force characteristics play an important role in in vivo muscle functioning. Hence, it is important to find a description that accounts for shortening induced alterations in these characteristics. The present study was undertaken to determine the dependency of post-shortening force decrease on shortening amplitude and muscle length for pennate rat medial gastrocnemius muscle (GM).

\section{Methods}

The experiments were performed on the medial gastrocnemius muscle of the rat $(G M)(n=6$, mean body mass: $294 \pm 5$ (SE) g). All contractions were induced by supramaximal stimulation of the Ischiadic nerve $(3 \mathrm{~mA}, 80$ $\mathrm{Hz}$ ). The experimental protocol consisted of a series of isokinetic contractions $(\mathrm{v}=10 \mathrm{~mm} / \mathrm{s})$ with different shortening amplitudes $(1-4 \mathrm{~mm})$ that ended at the same target length (Fig. 1a, b). Each isokinetic series was followed by a fully isometric contraction performed at the same muscle length. The protocol was repeated for a large number of target muscle lengths in the range of active muscle slack length and $4 \mathrm{~mm}$ over optimum muscle length. For each target length we calculated the difference $\left(\Delta \mathrm{F}_{\text {ma }}\right)$ between the isometric force redeveloped after the isokinetic shortening phase and the force of the fully isometric contraction. Muscle length $\left(l_{\mathrm{ma}}\right)$ was determined from photographs taken during the contractions.

\section{Results}

Figure 1 shows the experimental results for one muscle. In accordance with other studies (e.g. [1], [2]) we found that in rat GM post-shortening decrease in isometric force depends on shortening amplitude (Fig. 1b). For a given muscle target length, $\Delta F_{m a}$ increased linearly with shortening amplitude $\left(\Delta l_{\mathrm{ma}}\right)$. To incorporate the influence of shortening amplitude and muscle length on $\Delta F_{m a}$ into one single curve, we calculated the quotient between $\Delta \mathrm{F}_{\mathrm{ma}}$ and $\Delta \mathrm{l}_{\mathrm{ma}}$ for each 
contraction. These quotients were plotted against the average muscle lengths during the isokinetic shortening phase. All muscle lengths were expressed as deviations from the fully isometric optimum muscle length $\left(l_{\text {mao }}\right)$. The data for individual muscles are well described by a linear curve (Fig 1c). Regression analysis revealed a significant correlation between the data of individual muscles and a linear description. The positive slope of the curve corresponds to the observation that $\Delta \mathrm{F}_{\mathrm{ma}}$ is largest for shortening over optimum muscle length (e.g. [2]).

\section{Discussion}

The important result of the present study is that shortening induced alterations in the isometric length-force characteristics of rat GM can be described by a single linear curve (Fig. 1c). Note, that this curve only describes the influences of muscle length and shortening amplitude, the effect of shortening velocity is not incorporated in this curve. Marechal \& Plaghki [2] have shown that $\Delta \mathrm{F}_{\mathrm{ma}}$ is exponentially related to shortening velocity, with $\Delta \mathrm{F}_{\mathrm{ma}}$ being largest for slow shortening. They also showed that the influence of shortening velocity is independent of muscle
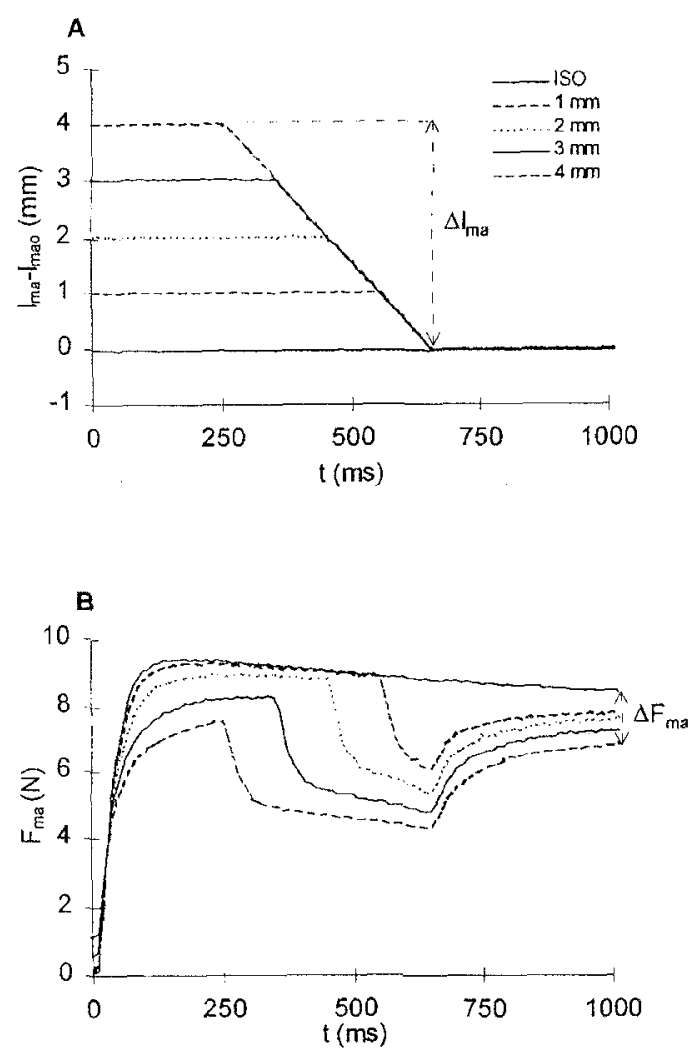

length. This indicates that the isometric length-force characteristics for a variety of shortening conditions can be described by two simple functions. A linear function describing the influence of both muscle length and shortening amplitude and an exponential function describing the influence of shortening velocity.

\section{References}

[1] Edman K.A.P., 1975, “ Mechanical deactivation induced by active shortening of isolated muscle fibres of the frog", J. Physiol., Vol 246, pp. 255-275.

[2] Marechal G. and Plaghki L., 1979, "The deficit of isometric tetanic tension redeveloped after a release of frog muscle at constant velocity", J. Gen. Physiol., Vol 73, pp. 453-467.

[3] Meijer K., Grootenboer H.J., Koopman H.F.J.M. and Huijing P.A., 1995, " The isometric length-force relationship during concentric contractions of the rat medial gastrocnemius muscle" In: Abstract Book Integrated Biomedical Engineering for Restoration of Human Function, ed. Feijen J., pp. 73-76. University of Twente, Enschede, The Netherlands.

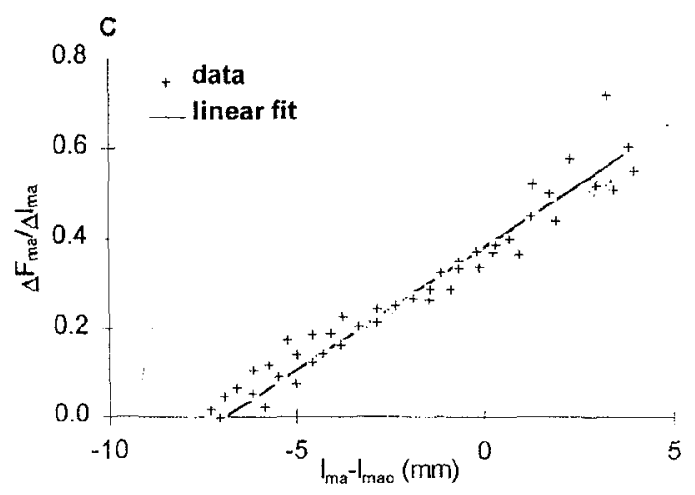

Figure 1. a) Isokinetic protocol (shortening amplitudes: $0-4 \mathrm{~mm}$, shortening velocity: $10 \mathrm{~mm} / \mathrm{s}$ ) for a given target muscle length. Muscle lengths are shown as a function of time and are expressed as deviations from the fully optimum muscle length $\left(\mathrm{l}_{\text {ma }}-\mathrm{l}_{\text {mao }}\right)$. The shortening amplitude $\left(\Delta \mathrm{l}_{\mathrm{na}}\right)$ of $4 \mathrm{~nm}$ is denoted by the arrow. b) Time-force trajectories, belonging to the isokinetic contractions represented in A). Post-shortening decrease in isometric force $\left(\Delta \mathrm{F}_{\mathrm{nu}}\right)$ after a shortening of $4 \mathrm{~mm}$ is denoted by the arrow. c) The muscle length dependency of the quotient $\Delta \mathrm{F}_{\text {imal }} / \Delta \mathrm{l}_{\text {ina. }}$. Experimental data is represented by the ' + ', the line represents a linear fit of the data $(r=0.98)$ 